\title{
Differentialdiagnose in der Augenheilkunde
}

\author{
Andreas Wedrich
}

Online publiziert: 14. Januar 2019

(C) Springer-Verlag GmbH Austria, ein Teil von Springer Nature 2019

Originalpublikation Burk A, Burk R, Kaden R (2018) Differenzialdiagnose in der Augenheilkunde - vom Befund zur Diagnose. gebunden, 520 Seiten, über 500 farbige Abbildungen., ISBN 978-3-942825-73-3, Preis: $€ 238$

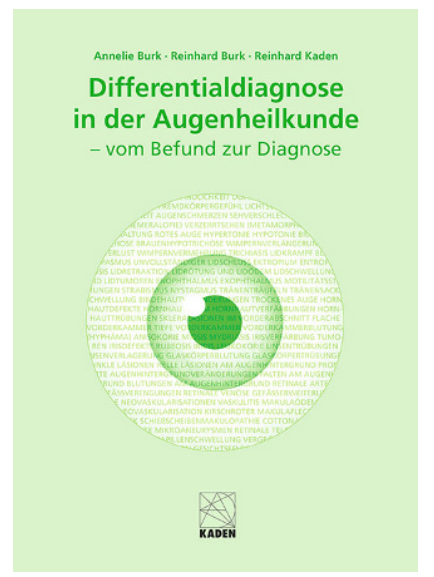

Das vorliegende Buch „Differenzialdiagnose in der Augenheilkunde - vom Befund zur Diagnose" der Autor*innen Burk, Burk und Kaden beschreitet in der Tat neue Wege in der Darstellung ophthalmologischer Krankheitsbilder und deren Differentialdiagnosen. Im Unterschied zu klassisch „systematischen“ Lehrbüchern, die nach Krankheitsbildern in den unterschiedlichen Organstrukturen gegliedert sind, folgt dieses Buch dem Ablauf eines klassischen Untersuchungsganges von der Anamnese bzw. den Symptomen über Befunde zur Diagnose.

Univ.-Prof. Dr. med. univ. A. Wedrich, FEBO ( $ه)$

Universitäts-Augenklinik, Medizinische Universität Graz, Auenbruggerplatz 4, 8036 Graz, Österreich andreas.wedrich@medunigraz.at
Schon im Inhaltsverzeichnis werden die einzelnen Begriffe (von „Asthenopie“ bis „Vaskulitis“) kurz spezifiziert. Es folgt nach nützlichen Anmerkungen zur Anamnese, Inspektion und Untersuchungen das erste Hauptkapitel „Symptome“, das die häufigsten und wichtigsten Symptome (wie z. B. Asthenopie, Blendempfindlichkeit, Schmerzen etc.) umfasst. Jedem Kapitel ist eine Kurzzusammenfassung der Differenzialdiagnosen des betroffenen Symptoms vorangestellt, die eine rasche Orientierung erlaubt. Danach folgt das eigentliche Kapitel mit Definition, typischen Begleitsymptomen und weiteren differenzialdiagnostischen Kriterien, die dem Leser einen Pfad zu den entsprechenden Diagnosen anbieten.

Anschließend folgt das zweite Hauptkapitel „Objektive Befunde“, das eine vergleichbare Gliederung aufweist. Wieder ist jedem Unterkapitel (wie z. B. Veränderung von Brauen und Lider, Veränderung der Vorderkammer, Veränderungen der Papille etc.) eine Kurzzusammenfassung der Differenzialdiagnosen in Form von Flussdiagrammen vorangestellt, bevor die Definition des Befundes und klassische Symptome sowie die einzelnen Diagnosen beschrieben werden, ergänzt durch ein ausführliches Glossar und Stichwortverzeichnis. Viele charakteristische klinische Bilder zu den einzelnen Themen tragen hervorragend zur Veranschaulichung des Textes und Wiedererkennen der Krankheitsbilder bei.

Dieses Buch besticht durch Übersichtlichkeit, Anschaulichkeit und die Möglichkeit zur raschen Orientierung, ausgehend von den Beschwerden, ist praxisnahe und ermöglicht durch seine Flussdiagramme und Beschreibungen eine rasche Eingrenzung der Diagnosen. Es wird dadurch dem Anspruch auf „neue Wege" gerecht und sollte in jeder Praxis oder Spitalsambulanz seinen Platz im Alltag finden.

Interessenkonflikt A. Wedrich gibt an, dass kein Interessenkonflikt besteht. 